\section{Single-cell cancer exomes}

Characterizing the mutations that occur during the evolution of tumors is a major challenge. Understanding whether cancers arise from monoclonal or polyclonal cells and determining the rate and progression of mutation accumulation are important areas of cancer research. Now, Jun Wang and colleagues report the first single-cell cancer exomes. Their first study (Cell 148, 873-885, 2012) reports exome sequencing of 82 bone marrow cells and 8 oral mucosal cells from a hematopoietic neoplasm called essential thrombocythemia (ET). The authors performed wholegenome amplification and sequenced targeted exome regions at $30 \times$ coverage. They calculated that the average allele dropout (where amplification failed in one allele) was $43 \%$, and the false discovery ratio was $6.0 \times 10^{-5}$. As a mutation found in only one cell is likely to be a false positive, only mutations present in at least five cancer cells are considered to be somatic mutations. The authors identified 712 somatic point mutations and performed principal-component analysis, which showed that the ET cells were distinct from the oral mucosal cells, suggesting a monoclonal origin for ET cells. The second study by these authors (Cell 148, 886-895, 2012) reports exomes of 25 cells from an individual with clear cell renal cell carcinoma.

\section{Tumor phylogenetics}

A solid tumor contains millions of cells. Understanding the origin and evolution of tumor cells can help in the development of effective treatments. To investigate intratumor heterogeneity with new genomic tools, such as exome sequencing, one can use a tour-de-force singlecell approach or undertake regional analysis. Charles Swanton and colleagues report a multiregional analysis of four primary renal carcinomas and associated metastases (N. Engl. J. Med. 366, 883-892, 2012). Using exome-sequencing data from nine regions of the nephrectomy specimens and metastases, they constructed tumor phylogenetic trees from two patients. In both tumors, the trees showed a branched evolution pattern and distinct metastasis clusters. Only one-third of somatic mutations were shared across all regions, whereas region-specific, 'private' mutations accounted for $22-53 \%$ of nonsynonymous point mutations and insertions and/or deletions. Analysis of histone marks suggested functional convergence, despite divergent mutations in the chromatin modification genes SETD2 and KDM5C. Genetic and functional heterogeneity was also observed for a mutation affecting mammalian target of rapamycin (mTOR) and its downstream effectors. This study sheds light on the mechanism of Darwinian tumor adaptation and on the potential for personalized medicine targeting mutations at the trunk of a tumor's phylogeny.

\section{Hematopoietic stem cell checkpoint}

Molecular checkpoints stop cells with genetic lesions from proliferating and spreading. Classical tumor suppressors, such as p53, exert pleiotropic effects on the cell cycle and apoptosis in response to DNA damage signals. It has been hypothesized that an unidentified checkpoint regulates stem cell self-renewal in adult tissues. Now, Lenhard Rudolph and colleagues report that the basic leucine zipper transcription factor BATF acts as a checkpoint factor for hematopoietic stem cell (HSC) self-renewal (Cell 148, 1001-1014, 2012). In an RNA

Written by Orli Bahcall, Pamela Feliciano, Wayne Peng \& Kyle Vogan interference screen, they found that mouse HSCs depleted of Batf continued contributing to hematopoiesis, despite having shortened telomeres, an aging-associated DNA lesion. Batf deletion or knockdown also improved HSC self-renewal in response to other types of DNA damage, such as $\gamma$-irradiation, although DNA breaks accumulated in these HSCs. Conversely, Batf expression was upregulated during aging or DNA damage in a cytokine signaling-dependent manner. Upregulation of Batf impaired HSC renewal, drove differentiation toward the lymphoid lineage and activated the classical tumor suppressor checkpoint proteins p53 and p21. In human HSCs isolated from individuals with myelodysplastic syndrome, BATF levels were positively correlated with expression of the cell cycle inhibitor CDKN1A and negatively correlated with telomere length.

$W P$

\section{Loss-of-function variants}

Daniel MacArthur and colleagues report a catalog of validated loss-offunction (LoF) variants predicted to completely disrupt human protein-coding genes (Science 335, 823-828, 2012). They identified 2,951 candidate LoF variants based on an analysis of whole-genome sequences of 185 samples from the pilot phase of the 1000 Genomes Project. They then computationally filtered this candidate list by taking into account local sequence context, gene annotation and read mapping and quality. Extensive experimental validation was performed, including genotyping with Illumina arrays and custom Sequenom assays. After manual reannotation, they report a list of 1,285 high-confidence LoF variants. Of this list, $32 \%$ were found to affect only a subset of the transcripts for the relevant gene, suggesting that functional proteins may still be produced. They estimate that there are $\sim 100 \mathrm{LoF}$ variants per human genome, with $\sim 20$ in a homozygous state. Genes with at least one LoF variant show less evolutionary conservation and have more paralogs, suggesting possible redundancy. The authors also imputed 417 of these LoF variants into Wellcome Trust Case Control Consortium (WTCCC) case-control datasets and found no excess of association signals compared to other protein-coding variants, suggesting that these variants have a minor role in common disease.

$O B$

\section{TTN mutations in cardiomyopathy}

Mutations in TTN, which encodes a very large sarcomeric protein titin, have been implicated in the etiology of numerous myopathies involving cardiac and skeletal muscle, but their role in dilated and hypertrophic cardiomyopathies has not been fully assessed. Christine Seidman and colleagues now report that truncating mutations in TTN are a common cause of dilated cardiomyopathy (N. Engl. J. Med. 366, 619-628, 2012). The authors used targeted capture and next-generation sequencing to analyze all annotated exons and splice sites of TTN in 203 individuals with dilated cardiomyopathy, 231 individuals with hypertrophic cardiomyopathy and 249 controls. They found a striking enrichment of truncating TTN variants in subjects with dilated cardiomyopathy (27\%) compared to those with hypertrophic cardiomyopathy ( $1 \%)$ or controls (3\%). Among familial cases of dilated cardiomyopathy, they observed strong co-segregation between truncating $T T N$ variants and clinical status. Unexpectedly, men carrying truncating TTN mutations had adverse cardiac events at significantly earlier ages than did women carriers. These results identify truncating mutations in TTN as the most common known genetic cause of dilated cardiomyopathy and could facilitate earlier diagnosis of the disease among carriers. 